Artículo científico

Volumen 31(3):649-661. Septiembre-diciembre, 2020

e-ISSN 2215-3608, doi:10.15517/am.v31i3.40289

http://www.revistas.ucr.ac.cr/index.php/agromeso

\title{
Riesgo económico en la producción de carne Angus y Hereford, La Posta, Chihuahua, México ${ }^{1}$
}

\section{Economic risk in the production of Angus and Hereford beef in La Posta, Chihuahua, Mexico}

\author{
Nicolás Callejas-Juárez ${ }^{2}$, Samuel Rebollar-Rebollar ${ }^{3}$,Eugenio Guzmán-Soria ${ }^{4}$, Aníbal Terrones-Cordero
}

1 Recepción: 13 de enero, 2020. Aceptación: 1 de abril, 2020. Este trabajo fue producto de un proyecto de investigación realizado en el Rancho Teseachic, La Posta, propiedad de la Universidad Autónoma de Chihuahua, México.

2 Universidad Autónoma de Chihuahua, Facultad de Zootecnia y Ecología. Periférico Francisco R. Almada, Km. 1. Chihuahua, Chihuahua, México. 31453.nicolascallejasjuarez@gmail.com (https://orcid.org/0000-0003-0170-1880).

3 Universidad Autónoma del Estado de México (UAEM), Centro Universitario UAEM Temascaltepec, Km. 67.5, Carretera Toluca-Tejupilco, Barrio de Santiago S/N. Temascaltepec, Estado de México, México. 51300. srebollarr@uaemex.mx; https://orcid.org/0000-0002-2906-0571

4 Instituto Tecnológico de Celaya, Posgrado en Administración. Av. Tecnológico y A. García Cubas s/n, Celaya, Guanajuato, México. 38010. eugenio.guzman@itcelaya.edu.mx (http://orcid.org/0000-0003-4713-7154).

5 Universidad Autónoma del Estado de Hidalgo, Instituto de Ciencias Económico Administrativas, Campus la Concepción, km. 2.5. San Juan Tilcuautla, San Agustín Tlaxiaca 42160 Hidalgo, México. aterrones68@hotmail.com (autor para correspondencia; http://orcid.org/00000002-2959-1807).

\section{Resumen}

Introducción. La preferencia al riesgo es la situación en la que un productor agropecuario decide invertir dinero y existe la probabilidad de obtener beneficios distintos a los deseados. Objetivo. Evaluar la preferencia al riesgo y la utilidad de la producción intensiva de carne de bovino Angus y Hereford en el Rancho Teseachic, La Posta, Chihuahua, México a través de la utilidad monetaria. Materiales y métodos. El experimento se realizó del 24 de marzo al 11 de agosto de 2017. Se evaluaron 52 bovinos, los cuales se alimentaron por un periodo de 141 días a base de maíz rolado $(53,3 \%)$ y granos de destilería $(37,8 \%)$. Los animales se dividieron en dos lotes: uno con 32 cabezas

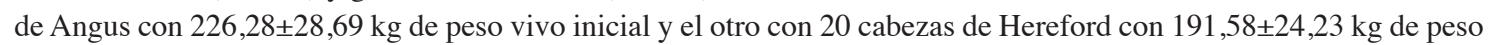
vivo. Se ajustó un modelo econométrico, mediante Mínimos Cuadrados Ordinarios, para determinar la preferencia al riesgo absoluta y relativa de la utilidad monetaria en la engorda de los bovinos. Resultados. Al final del periodo, el

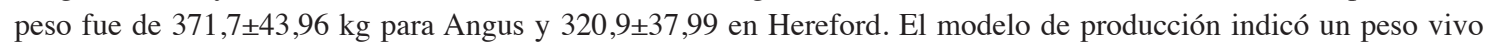
óptimo al sacrificio de 375,5 kg para Angus y 321,5 kg para Hereford. Conclusión. El productor rechazó la preferencia sin riesgo a la preferencia con riesgo. La producción de carne con terneros Angus presentó una menor preferencia por el riesgo $(20 \%)$ que en terneros Hereford (44\%) y por ende menor utilidad.

Palabras clave: utilidad monetaria máxima, productividad de los recursos, bioeconomía, econometría.

\section{Abstract}

Introduction. Risk preference is the situation in which an agricultural producer decides to invest money and there is a probability of obtaining benefits from those desired. Objective. To evaluate risk preference and utility of 
intensive Angus and Hereford beef production in Rancho Teseachic, La Posta, Chihuahua, Mexico through monetary utility. Materials and methods. The experiment was conducted from March 24th to August 11th 2017.52 cattle were evaluated and fed for a period of 141 days with rolled corn $(53,3 \%)$ and distillers' grain $(37,8 \%)$. The animals were divided into two lots: one with 32 Angus heads with $226.28 \pm 28.69 \mathrm{~kg}$ of initial live weight and the other with 20 Hereford heads with $191.58 \pm 24.23 \mathrm{~kg}$ of initial live weight. An econometric model was adjusted, using Ordinary Minimums Squares, to determine the absolute and relative risk preference of the monetary utility in cattle fattening. Results. At the end of the period, the weight was $371.7 \pm 43.96 \mathrm{~kg}$ for Angus and 320.9 \pm 37.99 for Hereford. The production model indicated an optimum live weight at slaughter of $375.5 \mathrm{~kg}$ for Angus and $321.5 \mathrm{~kg}$ for Hereford. Conclusion. The producer rejected the risk-free preference to the risky preference. Meat production with Angus calves showed a lower risk preference $(20 \%)$ than Hereford calves (44\%) and therefore less utility.

Keywords: maximum monetary utility, resource productivity, bioeconomy, econometrics.

\section{Introducción}

Los recursos humanos, monetarios y naturales representan el objeto de estudio de los profesionistas relacionados con los sistemas de producción. Una actividad importante de los economistas es maximizar el uso de los recursos utilizados en los procesos productivos. Sin embargo, su uso está sujeto a riesgos de tipo ambiental, mercado, entre otros. El riesgo se puede medir por la variabilidad en torno al valor esperado de la distribución de probabilidad de los retornos (Lee et al., 2010) o rendimiento de una inversión.

A menudo se dice que la producción agrícola es un negocio sujeto a riesgos, debido a lo complejo de los sistemas físicos y económicos, las decisiones y resultados de los agricultores son inciertos. Los resultados posibles, generalmente se asocian con un plan de acción o producción único (OECD, 2009). La introducción de opciones de productos básicos, mediante el esquema de contratos de futuros, amplió alternativas de comercialización para los productores de ganado y disminuyó el riesgo de mercado. Los productores de bovinos intentaban reducir el riesgo, asegurando precio de venta mediante la contratación futura o de la cobertura. Las estrategias de comercialización de opciones ofrecen fronteras de recompensa de riesgo no disponibles en los mercados tradicionales (Schroeder et al., 1989).

La preferencia al riesgo es uno de los supuestos más básicos del comportamiento económico. Pocos estudios han abordado la procedencia de las preferencias de riesgo y por qué difieren de un individuo a otro (Zhang et al., 2014), ya que los métodos tradicionales de estimación de funciones no consideran, en la medición el riesgo, el ambiente y el mercado.

$\mathrm{Al}$ respecto, uno de los métodos más utilizados para maximizar el uso de los recursos y reducir los riesgos son las funciones de producción, mismas que se obtienen en forma común a través del método de Mínimos Cuadrados Ordinarios (MCO) (Segura-Correa y Castellanos-Ruelas, 1999; Cienfuegos-Rivas et al., 2006; García-Muñiz et al., 2008). Sin embargo, presentan problemas de autocorrelación al utilizar una variable independiente relacionada con el tiempo, y violan los supuestos de independencia y consistencia de los estimadores (Wooldridge, 2010). Otro método no lineal, en sus variables, es el Cobb-Douglas que principalmente se utiliza de forma lineal (MoralesHernández et al., 2018). Existen otros métodos denominados robustos que eliminan el problema de la colinealidad en las variables independientes (Wooldridge, 2010; Guerrero y Melo, 2017); sin embargo, en algunos estudios se confirmó que evaluar el riesgo con la función de costo es semejante a evaluar la función de utilidad (Shankar, 2012; Iracheta-Lara et al., 2017). Estos consideraron, como evaluación estocástica, maximizar una función de producción 
mediante el producto marginal (PMg) o condición de primer orden; además, cuantificaron el riesgo de producción en términos de varianza del producto, al especificar la función de riesgo de tal manera que los insumos lo puedan aumentan o disminuir.

Una forma simple de evaluar el riesgo de un sistema de producción es el propuesto por Hidalgo et al. (2012) y Lefebvre y Vieider (2014), el cual consiste en utilizar la Eficiencia Estocástica Respecto a una Función (EERF), e implica comparar cada alternativa de utilidad monetaria con todas las otras alternativas simultáneamente, no por pares. Produce un conjunto eficiente más pequeño en el mismo rango de preferencias de riesgo, mediante un gráfico de semáforo (stoplight), y muestra la probabilidad de que un productor obtenga utilidad. En la producción pecuaria, la preferencia al riesgo implica que el productor no está dispuesto a perder dinero por la inversión realizada. Ante esto, el productor de carne bovina busca utilizar insumos adecuados con el fin de obtener carne de calidad que incentive a los consumidores a comprar el producto a mayor precio, obteniendo mayor utilidad y sin aumento de la inversión (Iracheta-Lara et al., 2017). Se espera que los inversores prefieran una riqueza futura esperada más alta a un valor más bajo y también, generalmente, son reacios al riesgo (Lee et al., 2010).

Un productor de carne de bovinos siempre tendrá una preferencia positiva hacia el riesgo, es decir, adverso al riesgo; por lo tanto, la función de utilidad con preferencia al riesgo será cóncava y la curvatura mide la afinidad al riesgo. En este tenor, Belasco et al. (2009) y Noussair et al. (2014) consideraron que la forma más adecuada de optimizar una función de producción es asociándole el riesgo. El riego mide la probabilidad de que un proceso productivo obtenga utilidad monetaria y que el administrador de un negocio no puede controlar, pero si prevenir, la eficiencia estocástica o simulación bajo riesgo ha sido analizada por Barham et al. (2014) y Lefebvre y Vieider (2014). Sin embargo, en México las investigaciones se han centrado en optimizar las funciones de producción deterministas (Hernández et al., 2011; Posadas et al., 2011; Ramírez et al., 2017; Morales-Hernández et al., 2018), dejando de lado la preferencia al riesgo de los productores; por ello, el objetivo de este trabajo fue evaluar la preferencia al riesgo y la utilidad de la producción intensiva de carne de bovino Angus (Aberdeen angus) y Hereford (Bos Taurus) en el Rancho Teseachic, La Posta, Chihuahua, México a través de la función de utilidad monetaria.

\section{Materiales y métodos}

El experimento denominado "pruebas de comportamiento" se realizó con 52 terneros (machos y hembras) en dos lotes nacidos en el Rancho Teseachic (La Posta), localizado a $28^{\circ}$ 53' 46,3" de latitud norte, 107' 27' 21,7" de longitud oeste y altitud de $1940 \mathrm{msnm}$, perteneciente al estado de Chihuahua, México, durante el periodo comprendido entre el 24 de marzo al 11 de agosto de 2017 (141 días). Para la comparación internacional, los valores monetarios se expresaron en dólares constantes de análisis (USD).

El primer lote se integró con dieciséis machos de la raza Angus, con peso vivo (PV) inicial y PV final de $226,3 \pm 5,6 \mathrm{~kg}$ y $371,8 \pm 7,8 \mathrm{~kg}$, respectivamente; el segundo lote se integró por nueve hembras y once machos de la raza Hereford, con PV inicial y final de $191,6 \pm 5,1$ y 321,3 $38,5 \mathrm{~kg}$, respectivamente. La alimentación fue ad libitum, con una sola dieta compuesta por maíz rolado (53,30\%), DDG $(37,8 \%)$, aceite vegetal $(1,9 \%)$, melaza $(5,0 \%)$, pre-mezcla de minerales $(0,5 \%)$, carbonato de calcio $(1 \%)$ y sal común $(0,5 \%)$. Los primeros 111 días, los bovinos se suplementaron con 1,2 $\mathrm{kg} \mathrm{día}^{-1}$ cabeza $^{-1}$, y los 30 restantes con $3,0 \mathrm{~kg} \mathrm{día}^{-1}$ cabeza ${ }^{-1}$. La dieta tuvo un costo promedio de $0,28 \mathrm{USD} \mathrm{kg}^{-1}$ (DOF 19,08 USD USD-1), durante los 141 días.

Para analizar el riesgo en el sistema de producción se estimó la función de utilidad monetaria $(\mathrm{G})$, que muestra una relación lineal entre el PV del animal (X) y el ingreso monetario (W) por la venta de carne. La función se obtuvo mediante el método de mínimos cuadrados ordinarios (MCO), utilizado por autores como Mballa y Sauceda (2018) y Morales-Hernández et al. (2018). La utilidad monetaria es la diferencia entre el ingreso $\left(\sum_{i=1}^{n} p_{i} q_{i}=Y\right.$ ) y el costo de producción $\left(\sum_{j=1}^{n} p_{j} q_{j}=X\right)$. 


$$
U=\sum_{i=1}^{n} p_{i} q_{i}-\sum_{j=1}^{n} p_{j} q_{j}
$$

La utilidad monetaria máxima o máximo técnico $\left(U^{*}\right)$ permite conocer el lugar geométrico en $\mathrm{W}$, donde al agregar unidades de insumo variable $\mathrm{X}$, la función es igual a cero; es decir, la utilidad marginal ( $U M g)$ muestra la elasticidad de la función de utilidad monetaria $(E U)$ respecto del PV.

$$
E U=\frac{\mathrm{dU}}{\mathrm{dX}} * \frac{X}{U}
$$

Esta forma determinística de optimizar una función está carente de riesgo, considerado elemento importante de un proceso productivo en el sector agropecuario. Un modelo simple de simulación estocástica tiene tres componentes: una variable dependiente (utilidad monetaria), una o más variables independientes sujetas a riesgo (costos variables), y una o más variables fijas (costos fijos). Los modelos de simulación estocástica se resuelven al utilizar un valor en $\mathrm{X}$ para generar una muestra de resultados para la variable dependiente $\mathrm{Y}$, al reconocer que $\mathrm{X}$ tiene riesgo (Richardson y Outlaw, 2008).

$$
Y=a+b X+\tilde{e}
$$

Donde ẽ representa la distribución de probabilidad del riesgo presente en el componente determinístico de Y, dado por $a+b X$; así, al considerar el riesgo que existe al pronosticar $Y_{t+1}$, entonces se debe utilizar una distribución de probabilidad en lugar de una estimación puntual.

Para seleccionar el mejor modelo de producción se utilizó el método robusto de mínimos cuadrados parciales (MCP) (Wold et al., 1984; Shankar, 2012). Los modelos se obtuvieron con el software SAS ${ }^{\circledR}$ 9.0, y para elegir el mejor modelo se utilizó el estadístico suma de cuadrados de predicción (PRESS).

\section{Análisis del riesgo}

En este trabajo, el riesgo se definió como la probabilidad de que un productor obtenga utilidad monetaria durante el periodo que duró el sistema de producción bajo estudio. Así, una de las formas más comunes de prevenir el riesgo es invertir en un seguro, donde la pregunta es ¿Cuánto invertir en un seguro?; sin embargo, el seguro solamente se cobra cuando la actividad productiva no puede ser realizada. Para el productor, lo más importante es tener argumentos económicos que permitan minimizar este riesgo. En los sistemas de producción pecuaria, el riesgo se asocia con maximizar la utilidad, por lo que es importante tener argumentos para atrasar o adelantar la finalización del proceso productivo. El mayor incentivo para asumir el riesgo es obtener una utilidad monetaria mayor a través del tiempo.

Un maximizador de utilidad esperada de preferencia al riesgo siempre aceptará una participación lo suficientemente pequeña en cualquier apuesta positiva de valor esperado cuando su función de utilidad sea diferenciable (Berk y Walden, 2013), y la decisión sobre el volumen de producción que debe producirse debe tomarse antes de la fecha de venta a la que se conocen los precios de mercado (Iracheta-Lara et al., 2017).

Si la inversión en la producción intensiva de carne bovina se correlaciona de forma positiva con la utilidad monetaria; entonces, su rendimiento esperado deberá ser mayor. En cambio, cuando existe una correlación negativa se le considera una inversión valiosa porque tendrá un rendimiento esperado menor a la tasa libre de riesgo y esto predispone al productor a tener aversión al riesgo. Si $C_{T}$ representa la preferencia en la producción intensiva de carne en $\mathrm{t}$ y $\pi_{t}$ la probabilidad de utilidad en $\mathrm{t}$, entonces la función de utilidad está dada por la siguiente ecuación: 


$$
U\left(C_{1}, C_{2}, \ldots, C_{T}, \pi_{1}, \pi_{2}, \ldots, \pi_{T}\right)=C_{1} \pi_{1}+C_{2} \pi_{2}, \ldots+C_{T} \pi_{T}
$$

Para el productor le es importante lo siguiente: ¿cuál es el precio que tendría la carne en el periodo t?, y ¿cuál será el PV óptimo del bovino? Al considerar dos periodos, la ecuación se reduce a comparar la utilidad más probable $\left(\pi_{1}\right)$ y la menos probable $\left(\pi_{2}\right)$, llamada utilidad media $(\hat{U})$, misma que se utilizó para generar los resultados de este trabajo.

$$
\widehat{U}=u\left(C_{1}\right) \pi_{1}+u\left(C_{2}\right) \pi_{2}
$$

Si $\pi_{1}$ (inicio del proceso productivo), entonces $\pi_{2}=1-\pi_{1}$ (fin del proceso productivo), esto significa que los dos momentos son mutuamente excluyentes; es decir, uno no depende del otro. La utilidad de invertir en el tiempo 1 puede representarse como $u\left(C_{1}\right)=a+b u$, y en el tempo 2 como $u\left(C_{2}\right)=a+b u$; sin embargo, solamente puede ocurrir una de las tres alternativas: $\sin$ riesgo $C_{0}$, que la utilidad sea mayor $\left(C_{2}\right)$ o que la utilidad sea menor $\mathrm{C}_{1}$; todo depende de la probabilidad en el precio de la carne de bovino en el mercado. En este caso, $C_{0}$ representa no invertir, $C_{1}$ la utilidad óptima, y $C_{2}$ utilidad con preferencia al riesgo.

La distribución del riesgo uniforme supone que una variable toma valores entre cero y uno, es decir, $X \sim(0$ a 1). La utilidad estocástica $(\tilde{\pi})$ es igual al valor estimado en forma determinista $(\hat{\pi})$ más el producto de la desviación estándar de la estimación determinista $(\hat{\sigma})$ multiplicada por el componente estocástico simulado (SND $=\operatorname{Norm}(0,1))$.

$$
\pi=\hat{\pi}+\hat{\sigma} * \operatorname{Norm}(0,1)
$$

El análisis de preferencia al riesgo tiene como base el desarrollado por Lambert y McCarl (1989). El modelo propone maximizar la función de utilidad monetaria $(\mathrm{U})$ sujeta a la restricción de riesgo (p) medida en desviaciones estándar (s).

$$
\operatorname{MaxE}(U)=\operatorname{Max}\left[\int_{-\infty}^{\infty} Y(x)-\int_{-\infty}^{\infty} C(x)\right]=\rho_{x} U\left(w_{x}\right)
$$

La función de utilidad muestra rendimientos decrecientes, por lo que significa que a mayor incremento del PV de los bovinos en engorda menor será la utilidad marginal (UMg) y mayor el riesgo (p). La función de utilidad (U) se expresa como la diferencia entre el ingreso $p f(y)$ y el costo de los recursos $w(x)$.

$$
U=f(w)=p f(y)-w(x)+e
$$

El valor máximo de la utilidad monetaria se obtiene cuando la función de utilidad marginal ( $U$ ') es igual a cero; esto es, la tasa de cambio en el ingreso por la venta de carne es igual a su costo de producción.

$$
U^{\prime}=f^{\prime}=\frac{d U}{d w}=0
$$

La segunda derivada ( $\left.U^{\prime \prime}\right)$ de U indica la tasa de cambio de la utilidad marginal. En una función de utilidad con un punto máximo, la segunda derivada siempre será negativa $\left(f^{\prime \prime}<0\right)$; por lo que se utiliza el valor absoluto y comparación de magnitudes.

$$
U^{\prime \prime}=f^{\prime \prime}=\frac{d U^{\prime}}{d w}
$$


La preferencia al riesgo se obtuvo por la segunda derivada de la función de utilidad y debe coincidir con la segunda etapa de la función de producción; por lo tanto, una función más cóncava tendrá mayor preferencia al riesgo y viceversa.

La preferencia al riesgo absoluto $\left(r_{A}\right)$ se definió como la tasa de cambio dada por el cociente de la segunda derivada de la función de utilidad ( $\left.U^{\prime \prime}\right)$ entre la función de la primera derivada de la función de utilidad ( $\left.U^{\prime}\right)$. En este sentido, un productor que tenga una preferencia absoluta creciente al riesgo si $r_{A}$ presenta rendimientos crecientes, preferencia constante al riesgo si los rendimientos son constantes y preferencia decreciente al riesgo si los rendimientos son decrecientes (Levy, 1969).

$$
r_{A}=-\frac{U^{\prime \prime}}{U^{\prime}}
$$

En tanto que la preferencia relativa al riesgo $\left(r_{R}\right)$ es igual al producto de la preferencia absoluta al riesgo absoluto y la cantidad producida (x).

$$
\begin{aligned}
r_{R}=x * r_{A}= & x *-\frac{U^{\prime \prime}}{U^{\prime}}=x *-\frac{U^{\prime \prime}(x)}{U^{\prime}(x)}=x * \frac{d U^{\prime}(x)}{d x} * \frac{x}{U^{\prime}(x)}=\frac{\frac{d U^{\prime}(x)}{U^{\prime}(x)}}{\frac{d x}{d x}} \\
= & \frac{\% U^{\prime}}{\% n x}
\end{aligned}
$$

Por definición, un productor tiene preferencia al riesgo cuando el valor esperado de la utilidad es igual acero, es decir, la suma del valor inicial (w) más el valor esperado $(z(w))$ es menor al valor inicial, $E(w+z)<w$. Es decir, un productor con preferencia al riesgo prefiere recibir una utilidad con mayor certeza, $E u(w+z) \leq U[w+E(z)]$.

Para evaluar el ingreso monetario se utilizaron los precios promedio proporcionados por el Sistema Nacional de Información e Integración de Mercados (SNIIM, 2017) de México, durante el periodo del proyecto. Al inicio del proceso productivo, el precio de la carne de bovino en pie fue $1,81 \$ \mathrm{~kg}^{-1}(\mathrm{w})$ y al finalizar el proceso de $1,95 \$$ $\mathrm{kg}^{-1}(\mathrm{E}(\mathrm{r}))$. Como el precio de la carne de bovino está sujeto a variaciones por la inflación, el ingreso fue evaluado a precios constantes (03 de febrero de 2017=100), mediante el uso del Índice Nacional de Precios al Consumidor (INPC) reportado por el INEGI (2017).

Al considerar $\pi$ como prima de riesgo, el valor de la preferencia al riesgo se determina por la suma de la probabilidad en el tiempo cero $(\mathrm{U}(w))$ y la probabilidad del premio al riesgo $\left(\mathrm{U}^{\prime}(w)\right)$.

$$
\begin{gathered}
U(w-\pi)=U(w)-\pi U^{\prime}(w) \\
E U(w+\tilde{z})=E\left[U(w)+z U(w)+\frac{1}{2} \tilde{r}^{2} U^{\prime \prime}(z)\right] \\
=U(w)+U^{\prime}(w) E(\tilde{r})+\frac{1}{2} U^{\prime \prime}(w) E\left(\tilde{r}^{2}\right) \\
E G(w+\tilde{z})=G(w)+\frac{1}{2} \sigma^{2} G^{\prime \prime}(w)
\end{gathered}
$$

Donde $E(z)=0$ y $\sigma^{2}=E\left(\tilde{z}^{2}\right)$. Asimismo, $\tilde{z}=\frac{1}{2} \sigma^{2} A(w)$, llamado el premio relativo al riesgo. Donde $A(w)=-\frac{U^{\prime \prime}(w)}{U^{\prime}(w)}$ representa el grado de concavidad de la función utilidad, mide la velocidad en que la utilidad 
marginal disminuye, también conocida como preferencia absoluta al riesgo (r). Esta indica la tasa a la cual la utilidad marginal disminuye por cada incremento unitario del precio de la carne de bovino en el mercado. Por el contrario, la aversión relativa al riesgo $(\mathrm{R})$ se mide a través de la elasticidad de la función marginal de utilidad:

$$
R=\frac{g U^{\prime}(w) / U(w)}{d w / w}=\frac{-w U^{\prime \prime}(w)}{U^{\prime}(w)}=w[r(w)]
$$

El productor deberá aceptar la preferencia al riesgo cuando $E U(w+\tilde{z})>U(w)$; esto quiere decir que el precio esperado de la carne, al finalizar el proceso productivo, deberá ser mayor que el que obtendría al inicio del proceso.

Para la obtención del riesgo absoluto y relativo de la función de utilidad monetaria, se utilizaron las funciones eficiencia estocástica con respecto a una función (SERF, por sus siglas en inglés) y luz de freno (stop light) del software Simetar (Hardaker et al., 2004). Fue considerado como valor mínimo de bienestar (w) el costo por kg del alimento, y como valor máximo de bienestar el $100 \%$ del valor inicial.

\section{Resultados}

\section{Ingreso con preferencia al riesgo}

El ingreso total del sistema de producción intensiva de carne de bovino, tanto de Angus como de Hereford, siempre fue creciente; sin embargo, la tasa de cambio del peso vivo (PV) de los animales mostró rendimientos marginales decrecientes (función de utilidad cóncava), por lo que la tasa de cambio de la utilidad marginal mostró este comportamiento. Esta cualidad, que muestran los sistemas de producción pecuarios, permitió decidir el momento óptimo en que debe finalizar el proceso productivo y maximizar la utilidad. Los puntos de mayor utilidad están dados por los picos positivos de la utilidad (días 51 y 68); asimismo, la peor opción se presentó en el día 17 (Figura 1).

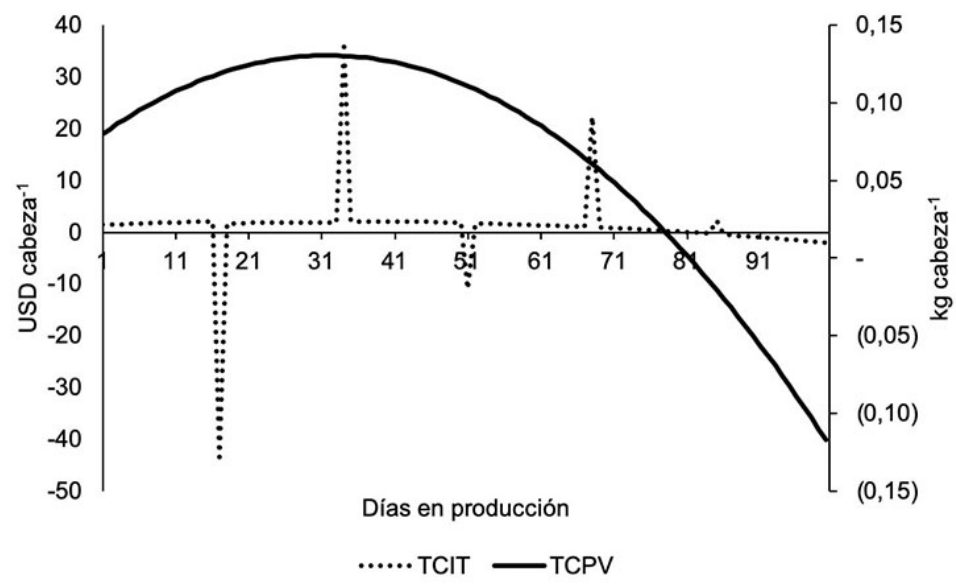

Figura 1. Peso vivo acumulado y consumo de alimento de bovinos Angus y Hereford en Namiquipa, Chihuahua, México, 2017.

TCIT: Tasa de Cambio del Ingreso Total; TCPV: Tasa de Cambio del Peso Vivo.

Figure 1. Accumulated liveweight and food consumption of Angus and Hereford cattle in Namiquipa, Chihuahua, Mexico, 2017. TCIT: Total Income Exchange Rate; TCPV: Live Weight Exchange Rate. 


\section{Optimización de la utilidad bajo riesgo}

Las funciones de utilidad para ambas especies de bovinos fueron cóncavas, lo que significa que el productor fue adverso al riesgo. En términos del sistema de producción, significa que los bovinos finalizados deberán tener un PV que permita obtener canales aceptadas por el mercado y por el mayor valor que tiene la carne al ser de mayor peso. La función de utilidad en bovinos Angus alcanzó su máximo con 414,25 kg de PV y una utilidad de 0,99 USD $\mathrm{kg}^{-1}$; mientras que para bovinos Hereford fue 383,66 kg de PV y 1,09 USD kg ${ }^{-1}$ (Figura 2).
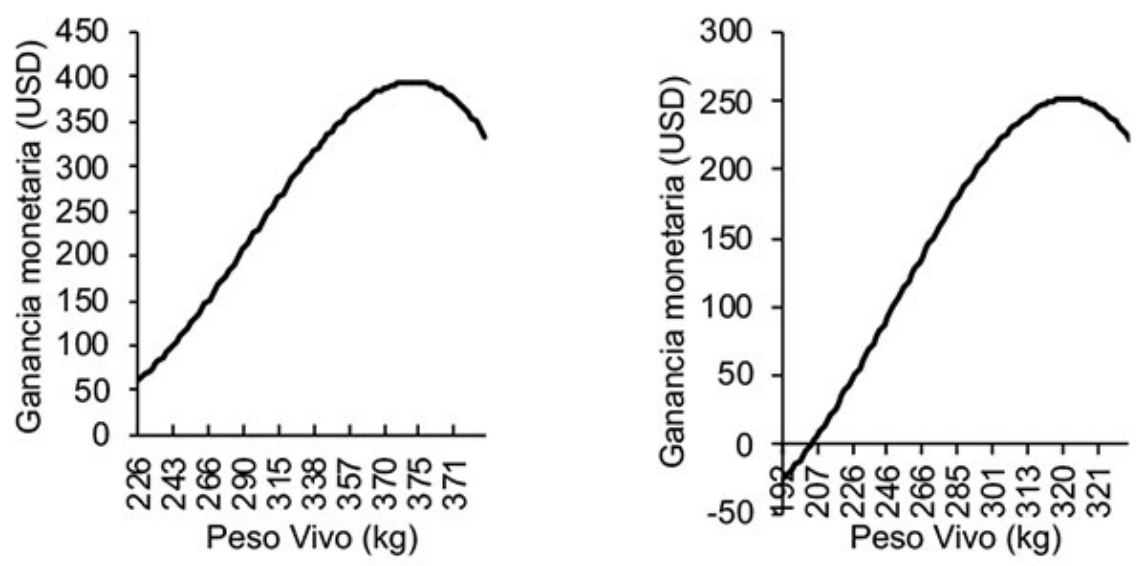

Figura 2. Funciones de ganancia monetaria para bovinos Angus y Hereford en Namiquipa, Chihuahua, México. 2017

Figure 2. Monetary gain functions for Angus and Hereford cattle in Namiquipa, Chihuahua, México. 2017.

\section{Preferencia al riesgo}

La utilidad monetaria por kilogramo producido en ganado Angus fue estadísticamente diferente $(\mathrm{p}<0,05)$ a la utilidad monetaria obtenida en ganado Hereford. Con una probabilidad del $95 \%$, el intervalo de confianza para la utilidad monetaria promedio fue $0,77-0,87 \$ \mathrm{~kg}^{-1}$ para carne Angus, y $0,45-0,56 \$ \mathrm{~kg}^{-1}$ para ganado Hereford; con una diferencia de $0,24-0,39 \$ \mathrm{~kg}^{-1}$. Sin embargo, el coeficiente de variación, como medida de la variabilidad del riesgo, fue mayor en ganado Hereford que en Angus (57,10>29,75); por lo que la utilidad monetaria por producir carne Angus fue menos riesgosa que producir carne Hereford (Figura 3).

La raza Angus mostró una probabilidad mayor de ganancia monetaria (menor riesgo) que la Hereford (más riesgosa). Esta diferencia de riesgo se atribuyó a la ganancia de PV. La producción de carne Angus tuvo una probabilidad de $80 \%$ de obtener una ganancia en el rango 0,57 hasta $1,05 \$ \mathrm{~kg}^{-1}$ (color verde), una probabilidad de $19 \%$ de obtener 0,28 a $0,55 \$ \mathrm{~kg}^{-1}$ (color amarillo) y una probabilidad de $1 \%$ de no tener utilidad (color rojo); mientras que la producción de carne Hereford tuvo una probabilidad de $56 \%$ de obtener una ganancia monetaria en el rango 0,57 a $0,79 \$ \mathrm{~kg}^{-1}$, una probabilidad de $20 \%$ de obtener de 0,28 a $0,55 \mathrm{USD} \mathrm{kg}^{-1} \mathrm{y}$ una probabilidad de $24 \%$ de perder a no obtener ganancia (Figura 4 ). 


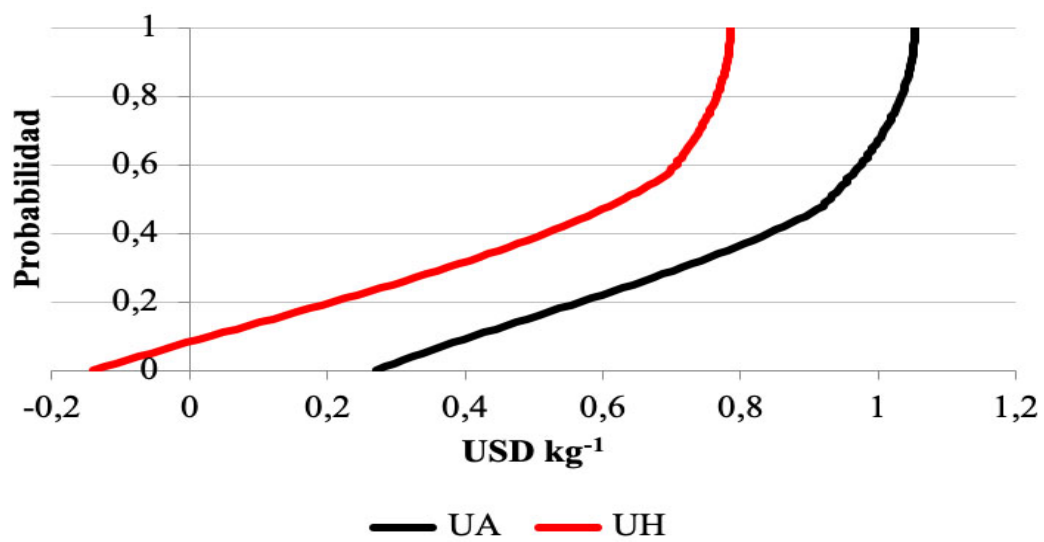

Figura 3. Utilidad monetaria acumulada de las razas Angus (UA) y Hereford (UH) en Namiquipa, Chihuahua, México. 2017.

Figure 3. Accumulated monetary utility of the Angus (UA) and Hereford (UH) races in Namiquipa, Chihuahua, Mexico. 2017.

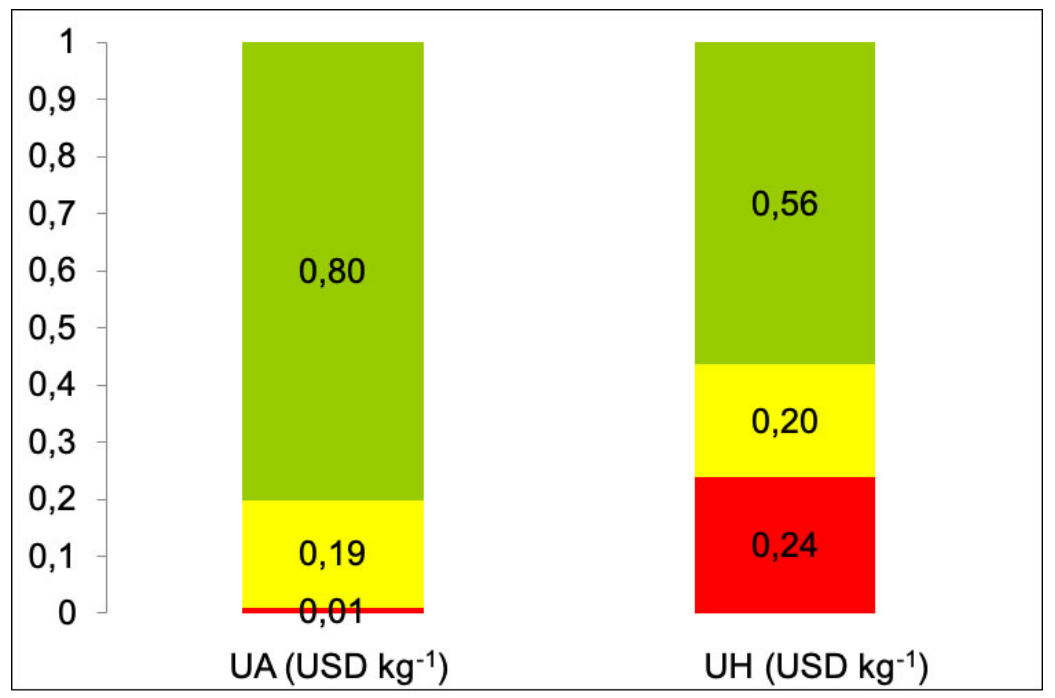

Figura 4. Probabilidad de utilidad monetaria de las razas Angus (UH) y Hereford (UH) en Namiquipa, Chihuahua, México. 2017.

Figure 4. Probability of monetary utility of the Angus (UA) and Hereford (UH) races in Namiquipa, Chihuahua, Mexico. 2017.

\section{Discusión}

Trabajos relacionados a los resultados fueron pocos; sin embargo, tales resultados se consideran válidos de replicarse en otros ambientes basados en el método utilizado. Así, en esta investigación, evaluar la asignación y productividad de los recursos en la economía agropecuaria conllevó a diferente resultado con el método clásico de costos e ingresos (contable) y con el método de preferencia al riesgo (económico-financiero). El primer método 
consideró que el valor de los recursos invertidos fue el mismo durante el tiempo, mientras que si se considera la preferencia al riesgo, el valor fue distinto porque se tomó en cuenta el costo de oportunidad de los recursos. En bovinos productores de carne, aun cuando la decisión del peso vivo (PV) al sacrificio depende de la tasa de crecimiento del animal, la tasa de interés y los precios de insumos y de la carne en pie está condicionada a que el animal sea alimentado por la ración óptima (Jarvis, 1974; Iracheta-Lara et al., 2017). Entonces, la preferencia (rechazo) al riesgo se convierte en la parte más importante de una inversión que no considera el productor agrícola y se manifiesta cuando la utilidad deseada es mayor, menor, o igual a la obtenida. La preferencia al riesgo se convierte en la expectativa no deseada de la inversión.

El sistema de producción intensivo de carne de bovino tuvo un límite superior dado por el PV final requerido por el mercado y se consideró adverso al riesgo (Belasco et al., 2009); esto significa que la función de utilidad fue cóncava y tomó la forma de la función de producción. Cuando un sistema de producción tiene una función de utilidad convexa se dice que el productor es atraído por el riesgo. Finalmente, si la función de utilidad es una línea recta entonces el productor es indiferente al riesgo.

La producción intensiva de bovinos de carne está sujeta a riesgo y la principal variable que lo explica es el precio del alimento balanceado; sin embargo, el productor minimiza o maneja el riesgo adquiriendo la cantidad total y en una sola exhibición de tal insumo, aun cuando tenga que asumir el costo de almacenamiento. El segundo es externo al proceso productivo y se encuentra en el precio de la carne. Durante el experimento, el intervalo de confianza para el precio promedio del alimento balanceado y del precio de la carne de bovino en pie fue de 0,26 a $0,46 \mathrm{USD} \mathrm{kg}^{-1}$ y 1,90 a 2,11 USD kg-1 , respectivamente, esto evidenció la importancia de conocer tanto la cantidad de alimento necesario para producir un $\mathrm{kg}$ de carne como el mercado de la misma. En esta investigación, el costo por kg de alimento balanceado fue mayor al mínimo del mercado, por lo que se tuvo un costo de oportunidad de $0,19 \mathrm{USD} \mathrm{kg}^{-1}$; a su vez, para el precio de venta de la carne en pie no hubo costo de oportunidad, esto significa que el productor vendió al precio máximo su producto.

La medición y análisis económico en el uso de recursos humanos, monetarios y naturales representa el insumo económico más importante para los productores de bienes y servicios al momento de tomar decisiones en cuanto al qué, cómo, cuándo, dónde, por qué y para quién producir. En el análisis de costos e ingresos de los procesos productivos se deben de considerar los costos del riesgo, ya que constituyen una variable importante en las decisiones de inversión de los productores. En el sector agropecuario de México, la preferencia al riesgo se considera como parte de los procesos productivos y carece de importancia para los productores. Estos riesgos podrían ser sistémicos o covariables; es decir, son comunes a grandes grupos de productores o podrían ser específicos de un individuo o idiosincrásicos (Ramaswami, 1992).

La evidencia inmediata de la asignación inadecuada de recursos son el problema de pérdida de productos y servicios, pérdida de la biodiversidad como consecuencia en el cambio de uso de suelo, proliferación de especies vegetales y animales no deseadas, y el consumo excesivo de recursos, productos y servicios. Aproximadamente, un $30 \%$ de los alimentos producidos para el consumo humano en todo el mundo se pierde o desperdicia cada año. Esto equivale a 1300 millones de toneladas de comida, 1 billón USD en costos económicos, cerca de 700 millones USD en costos ambientales y alrededor de 900 millones USD en costos sociales (Gustavsson et al., 2011). Esto significa que se están utilizando más recursos de los necesarios, por lo que una forma de resolver el problema es mediante la medición de la productividad de cada insumo o recurso; de tal manera que solamente se asigne la cantidad óptima de recursos bajo un concepto bioeconómico, es decir, al considerar los ciclos naturales de los recursos humanos, monetarios y naturales.

México consume alrededor de dos millones de toneladas de carne de bovino por año. Por tanto, para medir la productividad, considérese dos razas de ganado (Angus y Hereford), igual rendimiento de la canal (61 \%), igual costo de producción, igual precio de mercado, pero distinto peso vivo final (381 kg para Angus y $338 \mathrm{~kg}$ para Hereford). Si la decisión fuera utilizar la mejor raza, que en esta investigación fue la Angus, se necesitarían un millón 
de cabezas de ganado menos, equivalente a producir 2,8 millones de cabezas más o 254,4 miles de toneladas de carne más, que decidir utilizar la raza Hereford; entonces, la utilidad monetaria está en la productividad de los recursos.

\section{Conclusiones}

En este trabajo se comparó la preferencia al riesgo mediante una función de utilidad exponencial de dos razas de ganado bovino orientados a la producción intensiva de carne. El productor rechazó la preferencia sin riesgo en favor de la preferencia con riesgo; es decir, prefieren más que menos utilidad. La producción de carne con terneros Angus presentó una menor preferencia por el riesgo (20 \%) que en terneros Hereford (44\%).

Una variable que disminuyó el riesgo fue la eficiencia en la ganancia de peso, los terneros Angus tuvieron una ganancia de peso mayor que los terneros Hereford; estos últimos, necesitaron más días en producción para alcanzar el peso vivo óptimo, por lo que ante dos terneros de estas razas, siempre se debería elegir el Angus.

La función de utilidad permitió probar que, durante el ciclo productivo de las dos razas de bovinos carne que se evaluaron, la utilidad fue variable todos los días, debido al efecto de la ganancia de peso y al precio de la carne. La utilidad de los terneros Angus siempre fue mayor que los terneros Hereford y estuvo determinada por la ganancia de peso.

\section{Literatura citada}

Barham, B.L., J.P. Chavas, D. Fitz, V. Ríos, and L. Schechter. 2014. The roles of risk and ambiguity in technology adoption. J. Econ. Behav. Organization 97:204-218. doi:10.1016/j.jebo.2013.06.014

Belasco, E.J., M.R. Taylor, B.K. Goodwin, and T.C. Schroeder. 2009. Probabilistic models of yield, price, and revenue risks for fed cattle production. J. Agric. Appl. Econ. 41:91-105. doi:10.1017/S1074070800002571

Berk, J.B., and J. Walden. 2013. Limited capital market participation and human capital risk. Rev. Asset Pricing Stud. 3(1):1-37. doi:10.1093/rapstu/rat003

Cienfuegos-Rivas, E.G., M.A.R. D-Orúe-Ríos, M. Briones-Luengo, y J.C. Martínez-González. 2006. Estimación del comportamiento productivo y parámetros genéticos de características predestete en bovinos de carne (Bos taurus) y sus cruzas, VIII Región, Chile. Arch. Med. Vet. 38:69-75. doi:10.4067/S0301-732X2006000100010

García-Muñiz, J.G., E.G. Martínez-González, R. Núñez-Domínguez, R. Ramírez-Valverde, R. López-Ordaz, y A. Ruiz-Flores. 2008. Comparación de ecuaciones para ajustar curvas de lactancia en bovinos. Rev. Cient. FCV-LUZ 18(2):160-169.

Guerrero, S.C., y O.O. Melo. 2017. Una metodología para el tratamiento de la multicolinealidad a través del escalamiento multidimensional. Cienc. Desarrollo 8(2):9-24.

Gustavsson, J., C. Cederberg, U. Sonesson, R. Van-Otterdijk, and A. Meybeck. 2011. Global food losses and food waste. FAO, Rome, ITA.

Hardaker, J.B., J.W. Richardson, G. Lien, and K.D. Schumann. 2004. Stochastic efficiency analysis with risk aversion bounds: a simplified approach. Aust. J. Agric. Res. Econ. 48:2:253-270. doi:10.1111/j.1467-8489.2004.00239.x

Hernández, J., S. Rebollar, F.J. González, E. Guzmán, B. Albarrán, y A. García. 2011. La cadena productiva de ganado bovino en el sur del Estado de México. Rev. Mex. Agroneg. 29:672-680.

Hidalgo, C., M.P. Rodríguez, y R. Álvarez. 2012. El efecto de las subvenciones en la ganadería española: un análisis mediante un modelo de frontera estocástica. Pecvnia 14(ene-jun):93-106. doi:10.18002/pec.v0i14.594 
INEGI (Instituto Nacional de Geografía Estadística e Informática). 2017. Índice nacional de precios al consumidor. INEGI, Ciudad de México, MEX. https://www.inegi.org.mx/programas/inpc/2018/ (consultado 06 nov. 2019).

Iracheta-Lara, I.Z., E. Iglesias-Martínez, G. Méndez-Zamora, M.E. Esparza-Vela, y E. Santellano-Estrada. 2017. Modelado de riesgos para el sistema vaca-cría del estado de Chihuahua utilizando indicadores socioeconómicos y ambientales. Rev. Mex. Cien. Pecu. 8:193-200. doi:10.22319/rmcp.v8i2.4443

Jarvis, L.S. 1974. Cattle as capital goods and ranchers as portfolio managers: an application to the Argentine cattle sector. J. Political Econ. 82:489-520. doi:10.1086/260209

Lambert, D.K., and B.A. McCarl. 1989. Sequential modeling of white wheat marketing strategies. Appl. Econ. Perspect. Policy 11:105-115. doi:10.1093/aepp/11.1.105

Lee, C.F., J.E. Finnerty, and H.Y. Chen. 2010. Risk-aversion, capital asset allocation, and markowitz portfolio-selection model. In: C.F. Lee et al., editors, Handbook of quantitative finance and risk management. Springer, Boston, MA, USA. p. 69-92. doi:10.1007/978-0-387-77117-5_5

Lefebvre, M., and F.M. Vieider. 2014. Risk taking of executives under different incentive contracts: experimental evidence. J. Econ. Behav. Organizat. 97:27-36. doi:10.1016/j.jebo.2013.10.008

Levy, H. 1969. A utility function depending on the first three moments. J. Finance 24:715-719. doi:10.1111/j.1540-6261.1969. tb00395.x

Mballa, L.V., y A.Y. Sauceda. 2018. Análisis del hambre en el estado de Zacatecas bajo el modelo de Mínimos Cuadrados Ordinarios. Econ. Soc. Territorio XVIII(57):487-523. doi:10.22136/est01164

Morales-Hernández, J.L., F. J. González-Razo, y J. Hernández-Martínez. 2018. Función de producción de la ganadería de carne en la zona sur del Estado de México. Rev. Mex. Cien. Pecu. 9:1-13. doi.org/10.22319/rmcp.v9i1.4345

Noussair, C.N., S.T. Trautmann, and G. Kuilen. 2014. Higher order risk attitudes, demographics, and financial decisions. Rev. Econ. Stud. 81:325-355. doi:10.1093/restud/rdt032

OECD (Organization for Economic Co-operation and Development). 2009. Managing risk in agriculture: a holistic approach. OECD Publishing, Paris, FRA. doi:10.1787/9789264075313-1-en

Posadas, R., S. Rebollar, J. Hernández, F.J. González, A. Rebollar, y E. Guzmán 2011. Niveles de optimización económica en bovinos engordados en corral. En A.M. Arras-Vota, y O.A. Hernández-Rodríguez, editores, Administración, agrotecnología y redes de conocimiento. Pearson, MEX. p. 55-62.

Ramaswami, B. 1992. Production risk and optimal input decisions. Am. J. Agric. Econ. 74:860-869. doi:10.2307/1243183

Ramírez, J.I., A. Rebollar, S. Rebollar, B. Jaramillo, y F.J. González. 2017. Estudio de viabilidad económica para una engorda de bovinos en corral en el sur del estado de México. Rev. Mex. Agroneg. 41:742-753.

Richardson, J.W., and J.L. Outlaw. 2008. Ranking risky alternatives: innovations in subjective utility analysis. In: C.A. Brebbia, and E. Beriatos, editors, Risk analysis VI, WIT Transactions on Information and Communication Technologies series, Vol 39. Wit Press, GBR. p. 213-224. doi:10.2495/RISK080231

Schroeder, T.C., O.C. Grunewald, S.A. Langemeier, and D.M. Allen. 1989. An analysis of live cattle option hedging strategies. Agribusiness 5(2):153-168.

Segura-Correa, V., y A. Castellanos-Ruelas. 1999. Efecto de la suplementación fosforada sobre la utilidad de peso de bovinos en pastoreo en Yucatán, México. Vet. Méx. 30(3):257-261.

Shankar, S. 2012. Production economics in the presence of risk. Aust. J. Agric. Res. Econ. 56:597-620. doi:10.1111/j.14678489.2012.00600.x 
SNIIM (Sistema Nacional de Información e Integración de Mercados). 2017. Precio promedio ponderado de la leche. SNIIM, Ciudad de México, MEX. http://www.economia-sniim.gob.mx/2010prueba/PecComparativo.asp?var=Bov (consultado 4 nov. 2019).

Wold, S., A. Ruhe, H. Wold, and W.J. Dunn. 1984. The collinearity problem in linear regression. The partial least squares (PLS) approach to generalized inverses. J. Sci. Stat. Comp. 5:735-743. doi:10.1137/0905052

Wooldridge, J. 2010. Introducción a la econometría: un enfoque moderno. Cengage Learning Editores, Ciudad de México, MEX.

Zhang, R., T.J. Brennan, and A.W. Lo. 2014. The origin of risk aversion. PNAS 111:17777-17782. doi:10.1073/pnas.1406755111 